\title{
Über die Resorption pflanzlicher Sterine beim Menschen
}

\author{
Von \\ E. Böhle, E. Harmuth und M. Rajeivsky \\ Aus der I. Medizinischen Universitätsklinile Frankfurt/Main (Direktor: Prof. Dr. F. Hoff)
}

(Der Schriftleitung zugegangen am 8. August 1963)

\begin{abstract}
Mit Hilfe der Gaschromatographie wurde die Sterinzusammensetzung des Nüchternserums von 18 Gesunden mit normalen Blutfettwerten und 13 Arteriosklerotikern mit einer Hyperlipidämie bzw. Hypercholesterinämie untersucht. Bei annähernd $2 / 3$ der Probanden konnten neben dem Cholesterin kleinere Mengen an pflanzlichen Sterinen, wie $\alpha_{1}$-Sitosterin, Stigmasterin und $\beta$-Sitosterin nachgewiesen werden. Bei den Patienten ergaben sich durchweg niedrigere Phytosterinwerte als bei den Kontrollpersonen. - Weiter wurde der Einfluß peroraler Sitosteringaben auf die Gesamt-Lipide, den Cholesteringehalt und die Sterinzusammensetzung des Blutserums untersucht. In allen Fällen konnte eine geringe Zunahme der Gesamt-Phytosterine des Serums festgestellt werden, an der die einzelnen Phytosterinfraktionen in unterschiedlichem Maße beteiligt waren. Bei 8 Patienten mit Fettstoffwechselstörungen, denen über drei Monate täglich Sitosterin-Granulat oder polyensäurenreiche, phytosterinhaltige Pflanzenöle verabfolgt wurden, konnte zwar eine deutliche Senkung des Gesamt-Lipid- und Cholesterinspiegels im Verlauf der Beobachtungsperiode beobachtet werden, jedoch kein nennenswerter Anstieg des Phytosteringehalts im Blut.

Die vorliegenden Ergebnisse zeigen einerseits, daß der gesunde Organismus in der Lage ist, kleinere Mengen pflanzlicher Sterine zu resorbieren. Diese Fähigkeit scheint bei bestimmten Fettstoffwechselstörungen herabgesctzt oder aufgehoben zu sein. Andererseits läßt sich aus unseren Untersuchungen folgern, daß die blutcholesterinsenkende Wirkung pflanzlicher Sterine in erster Linie auf einer Hemmung der enteralen Cholesterinresorption beruht.
\end{abstract}

\begin{abstract}
The sterol composition of the fasting serum of 18 healthy individuals with normal blood fat values and of 13 atherosclerotics with hyperlipidaemia or hypercholesterolaemia was investigated by gas chromatography. Nearly ${ }^{2} / 3$ of the probands showed small amounts of plant sterols like $\alpha_{1}$-sitosterol, stigmasterol and $\beta$-sitosterol. All of the patients had a lower level of phytosterols than the controls. - Further, the influence of peroral doses of sitosterol on total lipids, cholesterol and the sterol composition of the serum was studied. In all cases, there was a small increase in the total phytosterols of the serum, with varying contributions from the individual phytosterol fractions. 8 patients with disturbances of fat metabolism were fed granulated sitosterol or plant oils rich in polyenoic acids and containing phytosterols daily for 3 months. There was a marked decrease in the levels of total lipids and cholesterol during the observation period, but no significant increase of phytosterols in the blood.

The results show that healthy persons can absorb small amounts of plant sterols. This ability seems to be decreased or abolished in certain disorders of fat metabolism. On the other hand, our results could indicate that the decrease of blood cholesterol caused by plant sterols is due primarily to the inhibition of the enteric absorption of cholesterol.
\end{abstract}

Die Erforschung des Sterinstoffwechsels beim Menschen unter physiologischen und pathologischen Bedingungen erstreckte sich bisher im wesentlichen auf das Cholesterin. Erst die Beobachtung von Petersen (1), daß die Zufuhr pflanzlicher Sterine im Tierexperiment eine blutcholesterinsenkende und antiatherosklerotische Wirkung ausübt, lenkte die Aufmerksamkeit auf diese dem Cholesterin chemisch sehr ähnlichen Verbindungen. Angesichts der Tatsache, daß man bei vielen Arteriosklerotikern Anomalien im Fettstoffwechsel, namentlich eine Hyperlipidämie oder Hypercholesterinämie nachweisen kann, lag es nahe, den Einfluß der Pflanzensterine auf das Serumcholesterin im menschlichen Organismus zu studieren. Pollack (2) beobachtete 1953 erstmalig beim Menschen einen cholesterinsenkenden Effekt von Sojasterinen. Ähnliche, teilweise auch widersprechende Ergebnisse wurden im Laufe der Jahre von zahlreichen anderen Autoren festgestellt (3-17) bzw. (18-22).

Der Wirkungsmechanismus pflanzlicher Sterine auf den Cholesterinstoffwechsel ist bisher noch ungeklärt. Der
Nachweis, daß pflanzliche Sterine die Resorption exogenen und endogenen Cholesterins beeinflussen $(23,24,25)$, führte zu der Vermutung, daß Phytosterine mit Cholesterin im Verdauungskanal schwer lösliche Mischkristalle bilden $(26,27)$ und auf diese Weise die Resorption des Cholesterins herabsetzen. Von Petersen und Mitarbeitern (28) sowie von BEST und DuncaN (29) konnte demgegenüber nachgewiesen werden, daß der blutcholesterinsenkende Effekt aufgehoben wird, wenn man beispielsweise Sitosterin als Ester verabreicht. Aus diesen Ergebnissen folgerten die Autoren, $\mathrm{da} B$ die Wirkung des Sitosterins auf einer kompetitiven Hemmung der Cholesterin-Esterbildung beim Resorptionsvorgang beruhe. Nach CHAIKOFF und Mitarbeitern (30) ist die Veresterung des Cholesterins mit Fettsäuren während des Transportes vom Darmlumen in die Lymphgefäße Voraussetzung für seine Resorption. Beide Theorien gehen davon aus, daß Pflanzensterine selbst vom Organismus nicht resorbiert werden, wie bereits früher SсHÖNHEIMER und Mitarbeiter (31-35) zeigen konnten. Demzufolge mußte angenommen 
werden, daß die Einwirkung des Sitosterins auf das Cholesterin vor der Resorption des letzteren in das Lymph- bzw. BlutgefäBsystem erfolgt. Auch in neueren tierexperimentellen Untersuchungen $(3,36,37,38)$ konnte teilweise mit Hilfe spezifischer Bestimmungsmethoden für pflanzliche Sterine nicht der Nachweis einer Resorption in die Blutbahn bzw. einer Einlagerung von Phytosterinen in verschiedene Organe erbracht werden.

Im Widerspruch zu diesen Ergebnissen beobachteten GoulD und Mitarbeiter (39) zunächst bei Ratten, daß nach Verfütterung von gleichen Mengen radioaktivem Sitosterin und Cholesterin der Anteil an resorbiertem Sitosterin $1 / 5-1 / 10$ des vom Körper aufgenommenen Cholesterins beträgt. Markiertes Sitosterin ersetzt 7\% des gesamten Lebercholesterins und 3,5\% des Cholesterins im übrigen Körper. - In Versuchen an 5 Menschen, die mit radioaktivem Sitosterin und Cholesterin behandelt wurden, fand Gousd (40), daß gemessen an der spezifischen Radioaktivität des Plasmas - Sitosterin und Cholesterin im Verhältnis 1:10 resorbiert wurden. Bilanzversuche am Menschen von SснӧN und HenNing (41) mit kristallinem $\beta$-Sitosterin und einer Sitosterinemulsion ergaben nach Anwendung der kristallinen Substanz eine negative Sterinbilanz, während nach Gaben von emulgiertem Sitosterin nur $30 \%$ der zugeführten Sterinmengen im Stuhl wiedergefunden wurden. Auch an Ratten.wurde von ScHöN und ENGELHARD (42) eine Sitosterinresorption beobachtet, die $50-60 \%$ der zugeführten Menge ausmachte. Aus diesen Ergebnissen folgerten SснӧN und Mitarbeiter (41, 42), daß im Gegensatz zur früheren Auffassung Pflanzensterine vom menschlichen Organismus resorbiert werden können, wenn auch in geringerem Maße als Cholesterin. Auf diese Weise sei Sitosterin auf Grund seiner chemischen Konfiguration, die sich von der des Cholesterins nur durch eine zusätzliche Äthylgruppe unterscheidet, in der Lage, die endogene Cholesterinsynthese durch Hemmung der hierzu erforderlichen Enzyme herabzusetzen. In Versuchen an der sog. experimentellen Fettleber konnten ScHöN und ENGELHARD (42) dementsprechend auch eine Verminderung der Cholesterinsyntheserate in der Leber der Versuchstiere nach Sitosteringaben feststellen. Diese Befunde stehen allerdings im Widerspruch zu den Ergebnissen von Gouln (40), der nach Sitosterinverfütterung keinen herabgesetzten Einbau von $\mathrm{C}_{14}$-Acetat in das Cholesterinmolekül feststellen konnte.

Die Unkenntnis der genaueren Zusammensetzung der Serumsterine beim Menschen muß angesichts dieser divergierenden Untersuchungsergebnisse über den Wir-。 kungsmechanismus pflanzlicher Sterine auf den Cholesterinstoffwechsel als ein Mangel empfunden werden, besonders deswegen, weil die Frage einer Beeinflussung des intermediären Cholesterinstoffwechsels durch Pflanzensterine den Nachweis dieser Verbindung im Blut voraussetzt. Hier eine Lücke auszufüllen, war das Ziel der vorliegenden Arbeit. Wir haben mit Hilfe der Gaschromatographie, die eine differenzierte Sterin- analytik zuläßt, Sterinanalysen des Blutserums bei Gesunden und Arteriosklerotikern ohne Vorbehandlung und nach Applikation pflanzlicher Sterine durchgeführt. Úber die Ergebnisse dieser Versuche soll hier berichtet werden.

\section{Methodik}

Bei 31 Probanden wurden Gesamt-Lipide, gesamtes und freies Cholesterin sowie die Sterinzusammensetzung des Nüchternblutserums untersucht. - Den Einfluß peroraler Sitosteringaben auf die Gesamt-Lipide, den Cholesteringehalt und die Sterinzusammensetzung des Blutes prüften wir bei insgesamt 16 Versuchspersonen. Hierfür standen zwei Zubereitungen zur Verfügung: eine 40-proz. Sitosterin-Suspension und Sitosteringranulat. Die Sterinzusammensetzung beider Präparate geht aus Abbildung 1 hervor.

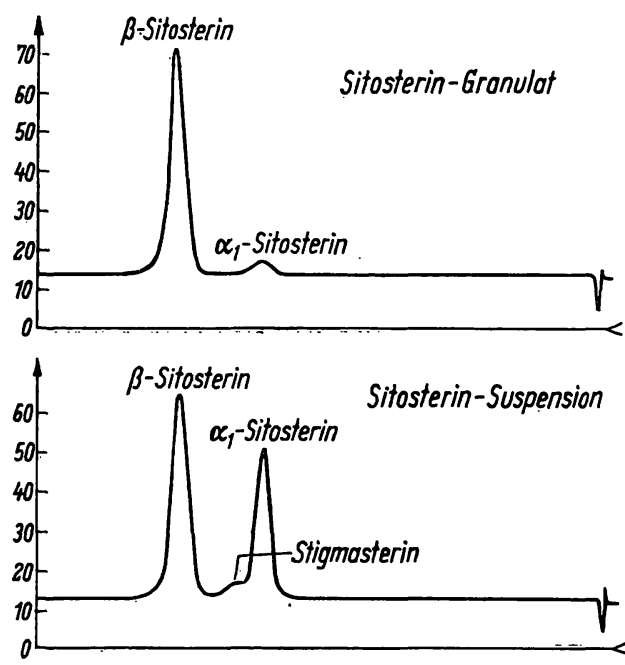

Abb. 1

Gaschromatographische Analysen der Sitosterinpräparate

\begin{tabular}{|c|c|c|c|}
\hline \multicolumn{4}{|c|}{ Zusammensetzung: } \\
\hline Sitoste & ulat & Sitosterin-S & \\
\hline $\begin{array}{l}\text { Sitosterin } \\
\text { Sitosterin }\end{array}$ & $\begin{array}{r}5,9 \% \\
94,1 \%\end{array}$ & $\begin{array}{l}\alpha_{1} \text {-Sitosterin } \\
\text { Stigmasterin } \\
\beta \text {-Sitosterin }\end{array}$ & $\begin{array}{r}36,8 \% \\
3,7 \% \\
59,5 \%\end{array}$ \\
\hline
\end{tabular}

Schließlich untersuchten wir die Sterinzusammensetzung des Blutes bei 8 Patienten, die im Rahmen medikamentöser oder diätetischer Maßnahmen über 3 Monate entweder Sitosteringranulat oder sitosterinhaltige Pflanzenkeimöle eingenommen hatten.

Sämtlichen Analysenergebnissen lagen Doppelbestimmungen zugrunde. Die Gesamt-Lipide wurden gravimetrisch aus $3 \mathrm{~m} /$ Blutserum nach Extraktion mit BLoorscher Lösung (43) (Alkohol : Äther $=3: 1$ ) und Reinigung (Chloroform) ermittelt. Die Bestimmung des gesamten und des freien Cholesterins erfolgte nach SCHÖNHEIMER und SpERRY (44) in der Modifikation von SPERRY und WEBB (45).

Für die Präparation der Sterine zur nachfolgenden gaschromatographischen Analyse wurden die beiden Gesamt-Lipidextrakte vereinigt und mit $10 \mathrm{~m} l$ einer 10-proz. methanolischen $\mathrm{KOH}$ 5 Stdn. auf dem Wasserbad verseift. Das Unverseifbare wurde $5 \mathrm{mal}$ mit Petroläther (Kp. $40^{\circ}$ ) ausgeschüttelt. Die vereinigten Petrolätherextrakte wurden mit Aqua dest. gewaschen und über Magnesiumsulfat getrocknet. Nach Eindampfen des Petroläthers wurden zur Chromatographie jeweils $15 \mu \mathrm{g}$ Gesamt-Sterine ein- 
gesetzt. Die Gaschromatographie der Sterine erfolgte im „PyeArgon"-Gaschromatographen") in Anlehnung an die von BeErTHUIS und Recourt (46) beschriebene Arbeitsweise: Säule $120 \times$ $0,4 \mathrm{~cm}, 5 \%$ Siliconöl auf „Chromosorb“ 80-100 mesh, Temperatur $240^{\circ}$ Druckabfall 2 atü, Detektorspannung 1500 Volt. Die Retentionszeiten der einzelnen Sterinfraktionen (Testsubstanzen) sind aus Abbildung 2 ersichtlich. Dic relativen Retentionszeiten sind in Tabelle 1 aufgeführt. $\alpha$-Tocopherol-Acetat, welches dieselbe Retentionszeit wic $\alpha_{1}$-Sitosterin besitzt, wird, wie wir festselben konnten, durch den Verseifungsvorgang zerstört.

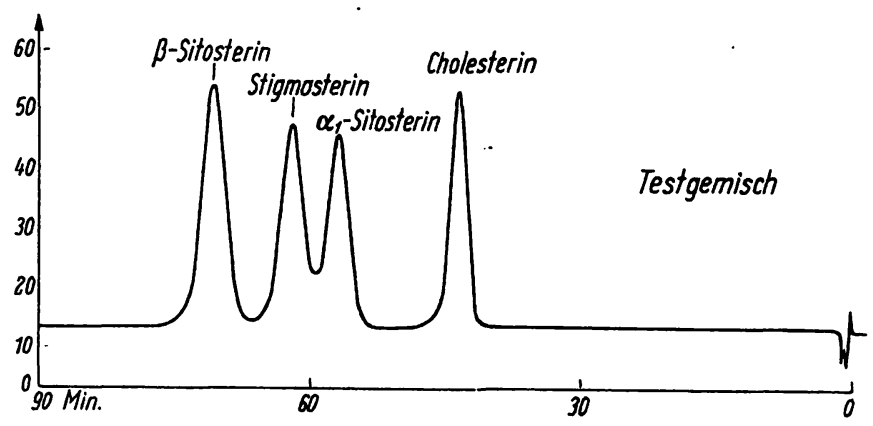

Abb. 2

Gaschromatographische Analyse eines Sterin-Testgemisches

Tab. 1

Relative Retentionszeiten der verschiedenen Phytosterine

\begin{tabular}{cc}
\hline Sterin & rel. Retentionszeit \\
\hline Cholesterin & 1,00 \\
$\alpha_{1}$-Sitosterin & 1,32 \\
Stigmasterin & 1,43 \\
$\beta$-Sitosterin & 1,64 \\
\hline
\end{tabular}

\section{Ergebnisse}

A. Es wurde zunächst die Sterinzusammensetzung des Nüchternserums von 31 Probanden untersucht. Hierbei handelte es sich um 18 klinisch gesunde Kontrollpersonen und um 13 Patienten mit einer manifesten Arteriosklerose. Bei der Kontrollgruppe lagen die Gesamt-Lipide des Blutserums zwischen 613 und $943 \mathrm{mg} \%$ und die GesamtCholesterinwerte zwischen 165 und $253 \mathrm{mg} \%$. Die Arteriosklerosepatienten wiesen Lipidkonzentrationen zwischen 900 und $1303 \mathrm{mg} \%$ und Cholesterinwerte zwischen 216 und $330 \mathrm{mg} \%$ auf.

Über die Zusammensetzung der Sterine des Blutserums wurde folgendes festgestellt (Tab. 2): Bei 7 der 18 Kontrollpersonen bestand das Unverseifbare ausschließlich aus Cholesterin, und bei 11 Gesunden ergab die gaschromatographische Analyse kleinere Mengen an Phytosterinen. Im Mittel enthielt das Unverseifbare des Nüchternserums der 18 Kontrollpersonen $98,60 \%$ Cholesterin und $1,40 \%$ Gesamt-Phytosterine. Bei den pflanzlichen Sterinen handelte es sich um $\alpha_{1-}^{-}, \beta$-Sitosterin und um Stigmasterin. Davon wurde am häufigsten, d. h. bei 8 Probanden, $\beta$-Sitosterin nachgewiesen. Die Konzentrationen erstreckten sich auf Werte zwischen $0,10 \%$ und $4,70 \%$ der Gesamt-Sterine. Eine isolierte $\beta$-Sito-

1) Leihgabe der Deutschen Forschungsgemeinschạft. sterinfraktion hatten 2 Gesunde, bei 4 Kontrollen konnte $\beta$-Sitosterin zusammen mit $\alpha_{1}$-Sitosterin nachgewiesen werden und bei 2 weiteren Probanden enthielt das Nüchternserum alle 3 Phytosterinfraktionen. Die Kombinationen von Stigmasterin und $\beta$-Sitosterin sowie von Stigmasterin und $\alpha_{1}$-Sitosterin wurden dagegen nicht beobachtet. 2 Gesunde wiesen ausschließlich Stigmasterin und ein weiterer eine isolierte $\alpha_{1}$-Sitosterinfraktion auf. Die Konzentrationen an $\alpha_{1}$-Sitosterin lagen zwischen 0,08 und $4,43 \%$ und an Stigmasterin zwischen 0,07 und $4,19 \%$. Beispiele für die verschiedenartigen Kombinationen der Sterinzusammensetzung des Nüchternblutes sind in Abbildung 3 aufgeführt. Insgesamt entfielen bei den 18 untersuchten Kontrollpersonen auf $\beta$-Sitosterin durchschnittlich $0,54 \%$ des Unverseifbaren, auf $\alpha_{1}$-Sitosterin $0,51 \%$ und auf Stigmasterin 0,35\%.

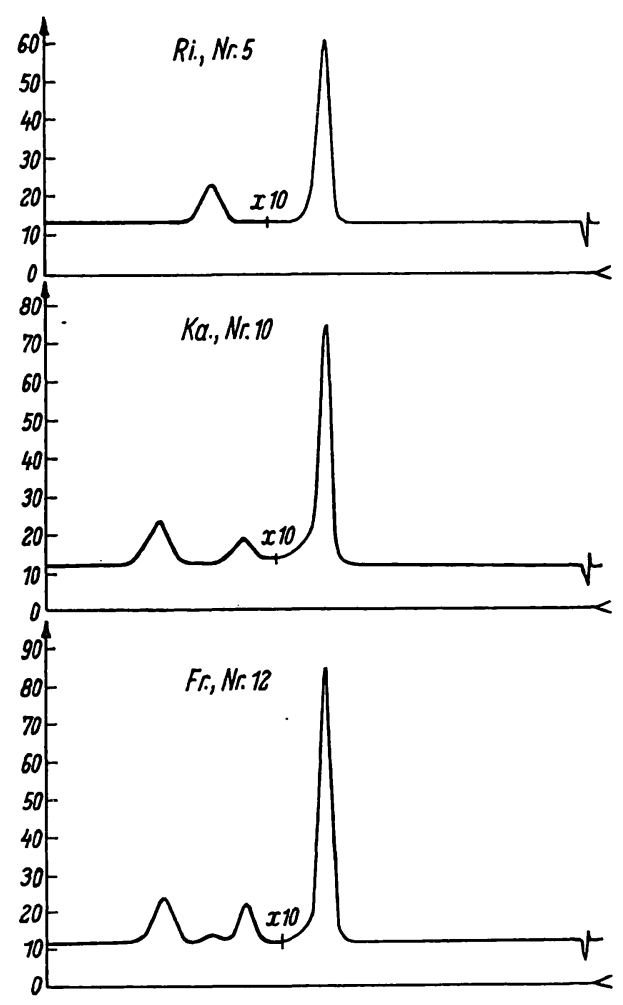

Abb. 3

Gaschromatographische Analysen der Sterine aus Nüchternserum von 3 Probanden ( $\times 10=10$-fache Verstärkung)

Unter den 13 Arteriosklerotikern, die in der Mehrzahl der Fälle eine deutliche Hyperlipidämie und Hypercholesterinämie aufwiesen, fanden sich 5 Patienten, bei denen das Unverseifbare des Nüchternserums ausschließlich aus Cholesterin bestand. Bei 8 Patienten wurden außerdem pflanzliche Sterine nachgewiesen. Im Mittel lag der Anteil der Gesamt-Phytosterine mit $0,21 \%$ erheblich niedriger als bei den Kontrollen $(1,40 \%)$. Es entfielen bei den Sklerosekranken durchschnittlich $99,79 \%$ des Unverseifbaren auf Cholesterin gegenüber $98,60 \%$ bei den Gesunden. Auch die $\mathrm{Zu}$ sammensetzung der Phytosterine ergab bei den Patienten ein anderes Bild als bei den Kontrollen. Am häufigsten 
Tab. 2. Gesamt-Cholesterin, Gesamt-Lipide und Sterinzusammensetzung des Nüchternserums von 18 gesunden Kontrollpersonen und 13 Arteriosklerotikern

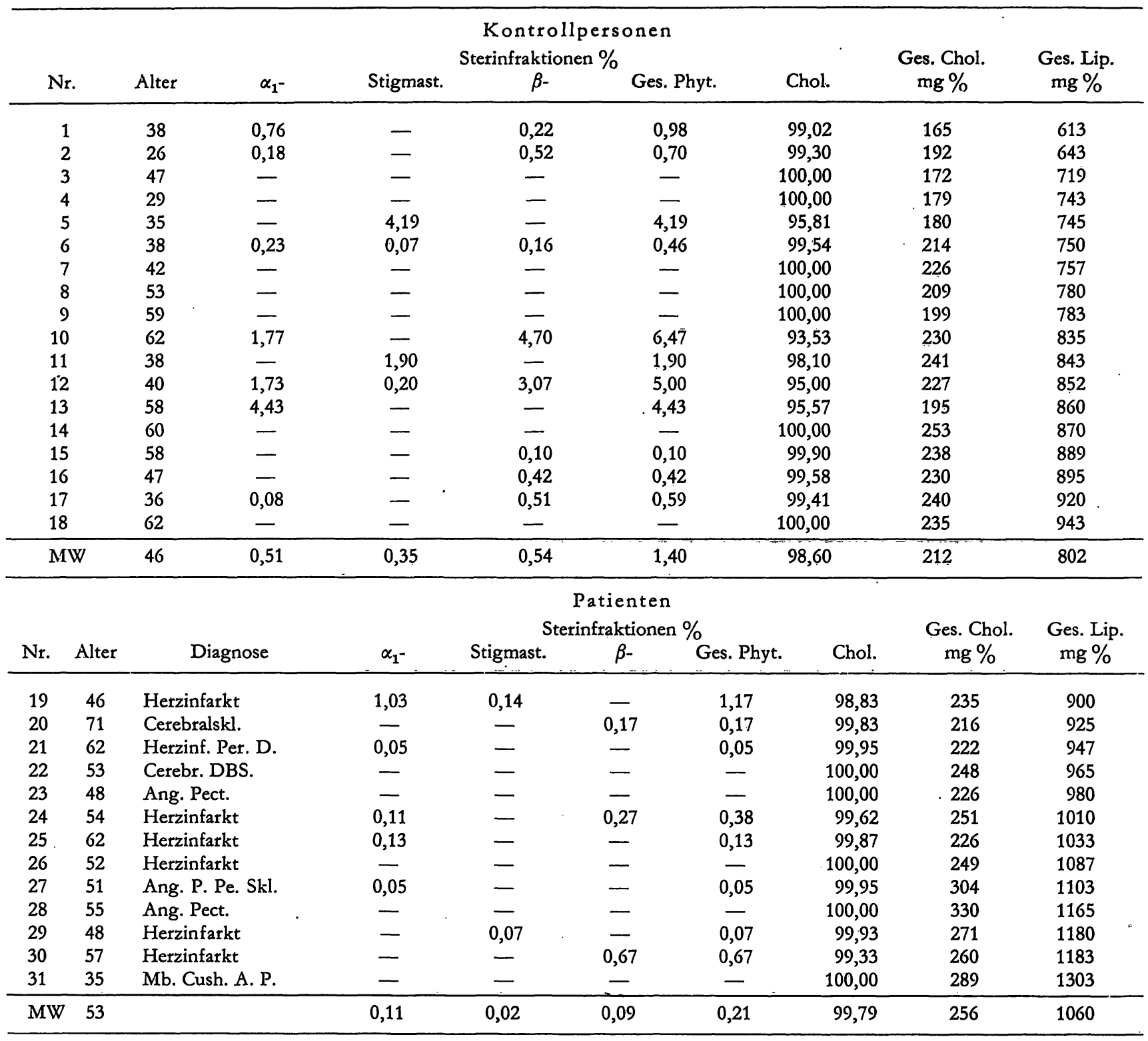

wurde bei den Arteriosklerotikern $\alpha_{1}$-Sitosterin nachgewiesen (5 Fälle), bei 3 Kranken enthielt das Unverseifbare $\beta$-Sitosterin und bei 2 weiteren Fällen Stigmasterin. Die Kombinationen von $\alpha_{1}$-Sitosterin und Stigmasterin bzw. $\alpha_{1}$-Sitosterin und $\beta$-Sitosterin lagen je einmal vor. Bei den übrigen Fällen kamen die einzelnen Phytosterinfraktionen jeweils isoliert zur Darstellung. Im Mittel errechnete sich bei den Patienten für $\alpha_{1^{-}}$ Sitosterin ein Wert von $0,11 \%$, für $\beta$-Sitosterin von $0,09 \%$ und für Stigmasterin von $0,02 \%$.

Trotz der beträchtlichen Unterschiede in der Sterin-。 zusammensetzung des Nüchternserums zwischen Gesunden und Patienten ergab sich für die Differenzen aller Mittelwerte infolge der starken Streuung der Einzelwerte keine statistische Signifikanz. Prüft man jedoch die Frage, ob der Gehalt an pflanzlichen Sterinen des Blutes bei unseren Probanden Beziehungen zur Höhe des Gesamt-Lipid- und Cholesterinspiegels aufweist, so gelangt man zu folgenden Feststellungen: Bei
Berücksichtigung der insgesamt 19 Kontrollpersonen und Patienten mit nachweisbaren Phytosterinanteilen im Blut errechnen sich die Korrelationskoeffizienten für Gesamt-Phytosterine (\%) zu Gesamt-Lipiden (mg\%) auf $-0,335$ und für Gesamt-Phytosterine (\%) zu Gesamt-Cholesterin (mg\%) auf - 0;316. Diese nahezu identischen Werte liegen allerdings noch innerhalb des Zufallbereiches. Die entsprechenden Regressionskoeffizienten belaufen sich auf $-0,004$ bzw. $-0,020, d$. h. bei einer Zunahme der Gesamt-Lipide um $100 \mathrm{mg} \%$ oder des Gesamt-Cholesterins um $20 \mathrm{mg} \%$ nimmt der Gesamt-Phytosterinanteil im Serum um 0,4\% ab.

B. Nachdem diese Ergebnisse gezeigt hatten, daß bereits unter physiologischen Bedingungen im Blutserum neben dem Cholesterin kleinere Anteile an pflanzlichen Sterinen enthalten sind, wurde die Frage geprüft, in welcher Weise sich die perorale Applikation von Sitosterin auf die Zusammensetzung der Serumsterine auswirkt. 
Tab. 3

Gesamt-Lipide, Gesamt-Cholesterin und Sterinzusammensetzung des Blutserums bei 8 Probanden im Verlaufe einer einmaligen Belastung mit $24 \mathrm{~g}$ Sitosterin

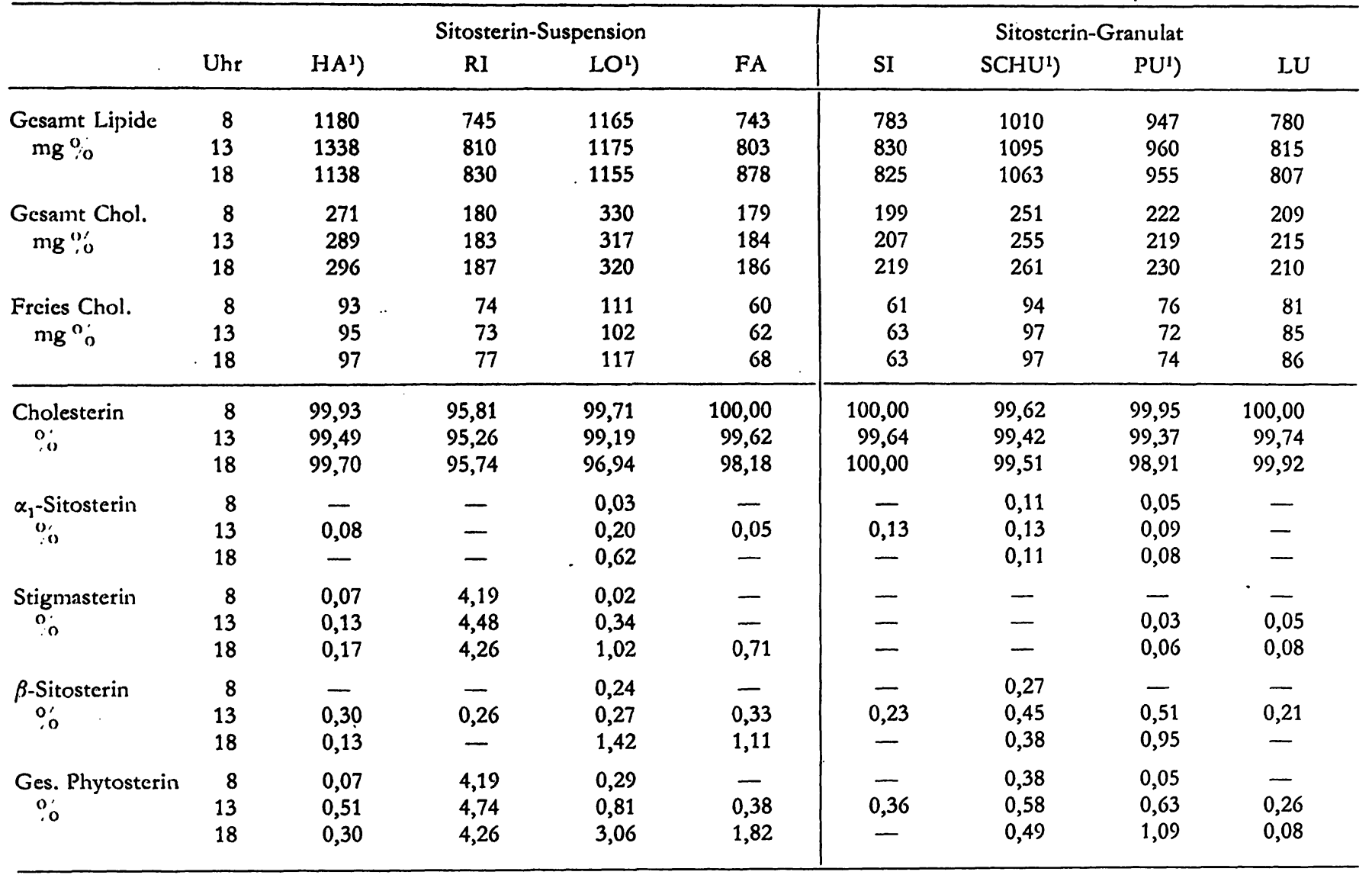

1) Arteriosklerotiker

Wir haben zu diesem Zweck zunächst bei 8 Versuchspersonen (4 Gesunde und 4 Arteriosklerotiker) je $24 \mathrm{~g}$ Sitosterin als Suspension oder Granulat vor dem Frühstück verabfolgt und nüchtern sowie nach 5 bzw. 10 Stunden die Zusammensetzung der Serumsterine untersucht. Wie aus Tabelle 3 hervorgeht fand in allen Fällen in geringem Umfange eine Resorption von pflanzlichen Sterinen statt. So kam es bei 5 Probanden zu einer Zunahme des prozentualen Anteils der Phytosterine, bei den restlichen 3 Versuchspersonen, deren Nüchternserum lediglich Cholesterin enthielt, traten zusätzlich in unterschiedlichem Ausmaß pflanzliche Sterine auf. Die Zunahme der Gesamt-Phytosterine erstreckte sich dabei auf Werte zwischen $0,20 \%$ und $2,77 \%$. Sie war bei denjenigen Probanden, die pflanzliche Sterine in Form der Suspension erhielten, durchweg stärker ausgeprägt als bei den anderen, denen Granulat verabfolgt wurde.

Was die einzelnen gaschromatographisch getrennten Sterinfraktionen anbetrifft, so fand sich beim $\alpha_{1}$-Sitosterin mit Ausnahme eines Falles im allgemeinen eine relativ geringe Anheburig nach 5 Stunden, obgleich die Suspension größere Mengen an $\alpha_{1}$-Sitosterin enthielt. Bei der Stigmasterinfraktion war bei 4 Probanden eine kontinuierliche, bis zur 10. Stunde nach Versuchsbeginn anhaltende Zunahme des prozentualen Anteils festzustellen. Diese Tatsache ist um so bemerkenswerter, als die Suspension nur sehr geringe Mengen dieses Sterins und das Granulat kein Stigmasterin enthielt. Eindeutiger waren die Konzentrationsänderungen des $\beta$-Sitosterinanteils. Diese Fraktion erfuhr bei allen Versuchspersonen eine deutliche Vermehrung. Bei 5 Fällen wurde ein Maximum des $\beta$-Sitosterinanstieges nach 5 Stunden beobachtet, bei den restlichen 3 Probanden hielt der Anstieg bis zur 10. Stunde p.c. an. Insgesamt ergibt sich aus den einzelnen Verlaufskurven in Abbildung 4, daß die Resorption von Phytosterinen bei den einzelnen Versuchspersonen sehr unterschiedlich erfolgte. Beziehungen $z$ wischen der Zunahme der Phytosterine im Blutserum nach peroraler Zufuhr und der Höhe des Phytosterinspiegels im Nüchternblut ließen sich nicht erkennen. Es bestanden auch keine Korrelationen zwischen dem Phytosterinanstieg nach Belastung und dem Gehalt des Nüchternserums an Gesamt-Lipiden oder Gesamt-Cholesterin.

C. In einer zweiten Serie wurde im Verlaufe einer 10-tägigen Beobachtungsperiode untersucht, inwieweit die tägliche Applikation von $18 \mathrm{~g}$ Sitosterin $(3 \times 6 \mathrm{~g}$ zu den Hauptmahlzeiten) eine Resorption von pflanzlichen Sterinen zur Folge hat und ob unter diesen Bedingungen die Sterinzusammensetzung des Nüchternserums nachhaltige Änderungen erfährt. Daneben haben wir die Auswirkung der Sitosterinbehandlung 


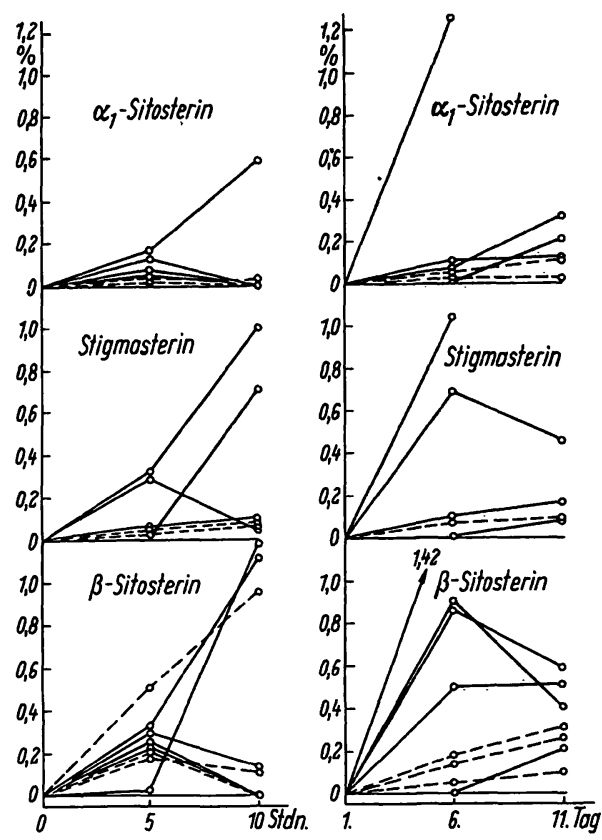

Abb. 4

Das Verhalten der einzelnen Pflanzensterine im Serum bei je 8 Probanden nach einmaliger und 10-tägiger Zufuhr von Sitosterin (O-O = Suspension, O-- - - O = Granulat) auf die Gesamt-Lipide und den Cholesteringehalt des Serums bei unseren Probanden verfolgt. Die Untersuchungen wurden än 4 gesunden Kontrollpersonen und 4 Arteriosklerotikern durchgeführt. Die Blutentnahmen erfolgten am 1., 6. und 11. Beobachtungstag. Wie Tabelle 4 zeigt, kam es im Verlaufe der Beobachtungsperiode bei 7 der 8 Probanden zu einer Abnahme der Gesamt-Lipide des Serums zwischen 50 und $169 \mathrm{mg} \%$ und bei 6 Fällen $z u$ einer Senkung des Gesamt-Cholesterinspiegels zwischen 15 und $40 \mathrm{mg} \%$. Es konnte bei sämtlichen Probanden eine Resorption pflanzlicher Sterine nachgewiesen werden. Auffällig war, daß bei Verabfolgung der Suspension bereits nach 5 Tagen relativ hohe Sitosterinkonzentrationen im Serum angetroffen wurden und daß eine weitere $\mathrm{Zu}$ nahme dẹ Gesamt-Phytosterine bis zum Versuchsende nicht eintrat (3 Fälle). Bei einem Patienten dieser Serie waren meßbare Phytosterinmengen erst nach 10 Tagen nachweisbar. - Ein etwas anderes Verhalten ergab sich nach Einnahme des Granulats. Hierbei konnte in allen Fällen eine kontinuierliche Zunahme des GesamtPhytosterins im Blut bis zum Ende der 10tägigen Beobachtungsperiode festgestellt werden. Allerdings lagen unter diesen Bedingungen die Phytosterinkon-

Tab. 4

Gesamt-Lipide, Gesamt-Cholesterin und Sterinzusammensetzung des Blutserums bei 8 Probanden nach täglich $18 \mathrm{~g}$ Sitosterin im Verlauf einer 10-tägigen Belastungsperiode

\begin{tabular}{|c|c|c|c|c|c|c|c|c|c|}
\hline & \multirow[b]{2}{*}{ Tag } & \multicolumn{4}{|c|}{ Sitosterin-Suspension } & \multicolumn{4}{|c|}{ Sitosterin-Granulat } \\
\hline & & WA & $\mathrm{KU}$ & $\mathrm{SCHN}^{1}$ ) & $\mathrm{KO}$ & $\left.\mathrm{SCHA}^{1}\right)$ & $\left.\mathrm{IM}^{1}\right)$ & GR & $\left.\mathrm{SCHE}^{1}\right)$ \\
\hline $\begin{array}{l}\text { Gesamt-Lipide } \\
\text { mg \% }\end{array}$ & $\begin{array}{r}1 \\
6 \\
11\end{array}$ & $\begin{array}{r}895 \\
845 \\
\times\end{array}$ & $\begin{array}{l}920 \\
888 \\
860\end{array}$ & $\begin{array}{l}965 \\
892 \\
870\end{array}$ & $\begin{array}{l}889 \\
770 \\
730\end{array}$ & $\begin{array}{l}1160 \\
1123 \\
1025\end{array}$ & $\begin{array}{l}1103 \\
1117 \\
1110\end{array}$ & $\begin{array}{l}719 \\
731 \\
657\end{array}$ & $\begin{array}{r}1033 \\
1012 \\
950\end{array}$ \\
\hline $\begin{array}{l}\text { Gesamt-Chol. } \\
\text { mg \% }\end{array}$ & $\begin{array}{r}1 \\
6 \\
11\end{array}$ & $\begin{array}{r}230 \\
211 \\
\times\end{array}$ & $\begin{array}{l}240 \\
220 \\
216\end{array}$ & $\begin{array}{l}248 \\
238 \\
214\end{array}$ & $\begin{array}{r}278 \\
-245 \\
241\end{array}$ & $\begin{array}{l}266 \\
251 \\
253\end{array}$ & $\begin{array}{l}304 \\
298 \\
264\end{array}$ & $\begin{array}{l}192 \\
190 \\
198\end{array}$ & $\begin{array}{l}226 \\
209 \\
209\end{array}$ \\
\hline $\begin{array}{l}\text { Freies Chol. } \\
\text { mg \% }\end{array}$ & $\begin{array}{r}1 \\
6 \\
11\end{array}$ & $\begin{array}{l}90 \\
80 \\
\times\end{array}$ & $\begin{array}{l}92 \\
89 \\
69\end{array}$ & $\begin{array}{l}92 \\
87 \\
83\end{array}$ & $\begin{array}{l}93 \\
92 \\
99\end{array}$ & $\begin{array}{r}103 \\
98 \\
99\end{array}$ & $\begin{array}{r}108 \\
93 \\
89\end{array}$ & $\begin{array}{l}74 \\
77 \\
84\end{array}$ & $\begin{array}{l}x \\
x \\
x\end{array}$ \\
\hline $\begin{array}{l}\text { Cholesterin } \\
0 \% \\
\%\end{array}$ & $\begin{array}{r}1 \\
6 \\
11\end{array}$ & $\begin{array}{c}99,58 \\
94,86 \\
\times\end{array}$ & $\begin{array}{l}99,41 \\
98,34 \\
98,47\end{array}$ & $\begin{array}{r}100,00 \\
99,14 \\
. \quad 99,20\end{array}$ & $\begin{array}{r}99,90 \\
98,60 \\
98,80\end{array}$ & $\begin{array}{r}100,00 \\
100,00 \\
99,71\end{array}$ & $\begin{array}{l}99,95 \\
99,85 \\
99,83\end{array}$ & $\begin{array}{r}100,00 \\
99,75 \\
99,60\end{array}$ & $\begin{array}{l}99,87 \\
99,67 \\
99,49\end{array}$ \\
\hline $\begin{array}{l}\alpha_{1} \text {-Sitosterin } \\
\% \\
\%\end{array}$ & $\begin{array}{r}1 \\
6 \\
11\end{array}$ & $\begin{array}{l}\overline{1,25} \\
\times\end{array}$ & $\begin{array}{l}0,08 \\
0,15 \\
0,44\end{array}$ & $\overline{-}$ & $\begin{array}{l}\overline{0,11} \\
0,13\end{array}$ & - & $\begin{array}{l}0,05 \\
0,08 \\
0,07\end{array}$ & $\overline{-}$ & $\begin{array}{l}0,13 \\
0,19 \\
0,25\end{array}$ \\
\hline $\begin{array}{l}\text { Stigmasterin } \\
\% \%\end{array}$ & $\begin{array}{r}1 \\
6 \\
11\end{array}$ & $\begin{array}{c}- \\
1,05 \\
\times\end{array}$ & $\begin{array}{l}- \\
0,10 \\
0,17\end{array}$ & $\frac{-}{-}$ & $\begin{array}{l}- \\
0,69 \\
0,46\end{array}$ & $\overline{-}$ & $\frac{-}{-}$ & $\begin{array}{l}- \\
0,07 \\
0,09\end{array}$ & $\frac{-}{-}$ \\
\hline $\begin{array}{l}\beta \text {-Sitosterin } \\
\% \\
\%\end{array}$ & $\begin{array}{r}1 \\
6 \\
11\end{array}$ & $\begin{array}{l}0,42 \\
1,84 \\
\times\end{array}$ & $\begin{array}{l}0,51 \\
1,41 \\
0,92\end{array}$ & $\begin{array}{l}- \\
0,86 \\
0,59\end{array}$ & $\begin{array}{l}0,10 \\
0,60 \\
0,61\end{array}$ & $\overline{-}$ & $\begin{array}{l}\overline{0} \\
0,05 \\
0,10\end{array}$ & $\begin{array}{l}- \\
0,18 \\
0,31\end{array}$ & $\begin{array}{l}\overline{0,14} \\
0,26\end{array}$ \\
\hline $\begin{array}{l}\text { Ges. Phytosterin } \\
\%\end{array}$ & $\begin{array}{r}1 \\
6 \\
11\end{array}$ & $\begin{array}{c}0,42 \\
5,14 \\
\times\end{array}$ & $\begin{array}{l}0,59 \\
1,66 \\
1,53\end{array}$ & $\begin{array}{l}- \\
0,86 \\
0,80\end{array}$ & $\begin{array}{l}0,10 \\
1,40 \\
1,20 .\end{array}$ & $\frac{-}{0,29}$ & $\begin{array}{l}0,05 \\
0,13 \\
0,17\end{array}$ & $\begin{array}{l}\overline{0,25} \\
0,40\end{array}$ & $\begin{array}{l}0,13 \\
0,33 \\
0,51\end{array}$ \\
\hline
\end{tabular}

1) Arteriosklerotiker 
zentrationen des Serums auch nach 10 Tagen noch deutlich niedriger als bei denjenigen Probanden, welche die Suspension erhalten hatten.

Die einzelnen Phytosterinfraktionen reagierten unterschiedlich (Abb. 4). Der $\alpha_{1}$-Sitosterinanteil nahm bei 5 der 8 Versuchspersonen nur leicht $z u$, lediglich bei einem Probanden kam es zu einem deutlichen Anstieg. Im allgemeinen war die Zunahme dieser Fraktionen nach Applikation der Suspension etwas stärker ausgeprägt, als nach Verabfolgung des Granulats. Ein Anstieg des Stigmasterinanteils konnte bei 2 Probanden recht deutlich, bei 3 weiteren nur in geringem Umfang festgestellt werden. Regelhafter war, wie aus Abbildung 4 hervorgeht, die Vermehrung des $\beta$-Sitosterins, die bei allen 8 Versuchspersonen nachzuweisen war und die wiederum bei Zufuhr der Suspension stärker in Erscheinung trat als beim Granulat. Auch in dieser Versuchsserie ergaben sich bei den Probanden keine Beziehungen zwischen der Höhe der Gesamt-Lipide, des Gesamt-Cholesterins oder des Nüchternspiegels an pflanzlichen Sterinen einerseits und dem Anstieg der Phytosterine des Blutes andererseits. Auch eine Relation zwischen der Senkung des Gesamt-Lipid-und Cholesterinspiegels und der Zunahme der Phytosterine im Blutserum konnte nicht festgestellt werden.
D. Wir haben schließlich Untersuchungen an 8 Patienten vorgenommen, denen über 3 Monate täglich Sitosterin entweder als Granulat oder in kleineren Mengen im Rahmen einer diätetischen Therapie mit polyensäurenreichen Ölen zugeführt wurde.

Der Phytosteringehalt der verwendeten polyensäurenreichen Präparate und die prozentuale Zusammensetzung der pflanzlichen Sterine geht aus Abbildung 5 hervor. In Tabelle 5 sind die Ergebnisse der Blutanalysen vor und nach 3-monatiger Behandlung zusammengestellt. Es geht daraus hervor, daß in keinem Fall vor Einnahme der verschiedenen Präparate nachweisbare Phytosterinmengen im Blut vorhanden waren. Bei allen Patienten konnte zwar eine Senkung des Gesamt-Lipid- und Cholesterinspiegels durch die verschiedenen diätetischen Maßnahmen erreicht werden, die Sterinzusammensetzung des Blutes blieb jedoch in den meisten Fällen unbeeinflußt. Es heben sich hier besonders die beiden Xanthomatosekranken heraus, bei denen trotz Einnahme von mehr als $500 \mathrm{~g}$ pflanzlicher Sterine während des gesamten Beobachtungsraumes keine meßbaren Phytosterinmengen im Blut auftraten. Auch unter den Bedingungen einer Ernährung mit polyensäurenreichen Pflanzenölen, welche kleinere Mengen Phytosterinanteile enthalten, stieg der GesamtPhytosteringehalt des Blutes in 3 Fällen nur wenig an.

Tab. 5

Gesamt-Lipide, Gesamt-Cholesterin und Sterinzusammensetzung des Blutserums von 8 Patienten nach Behandlung mit SitosterinGranulat oder polyensäurenreichen, phytosterinhaltigen Pflanzenölen

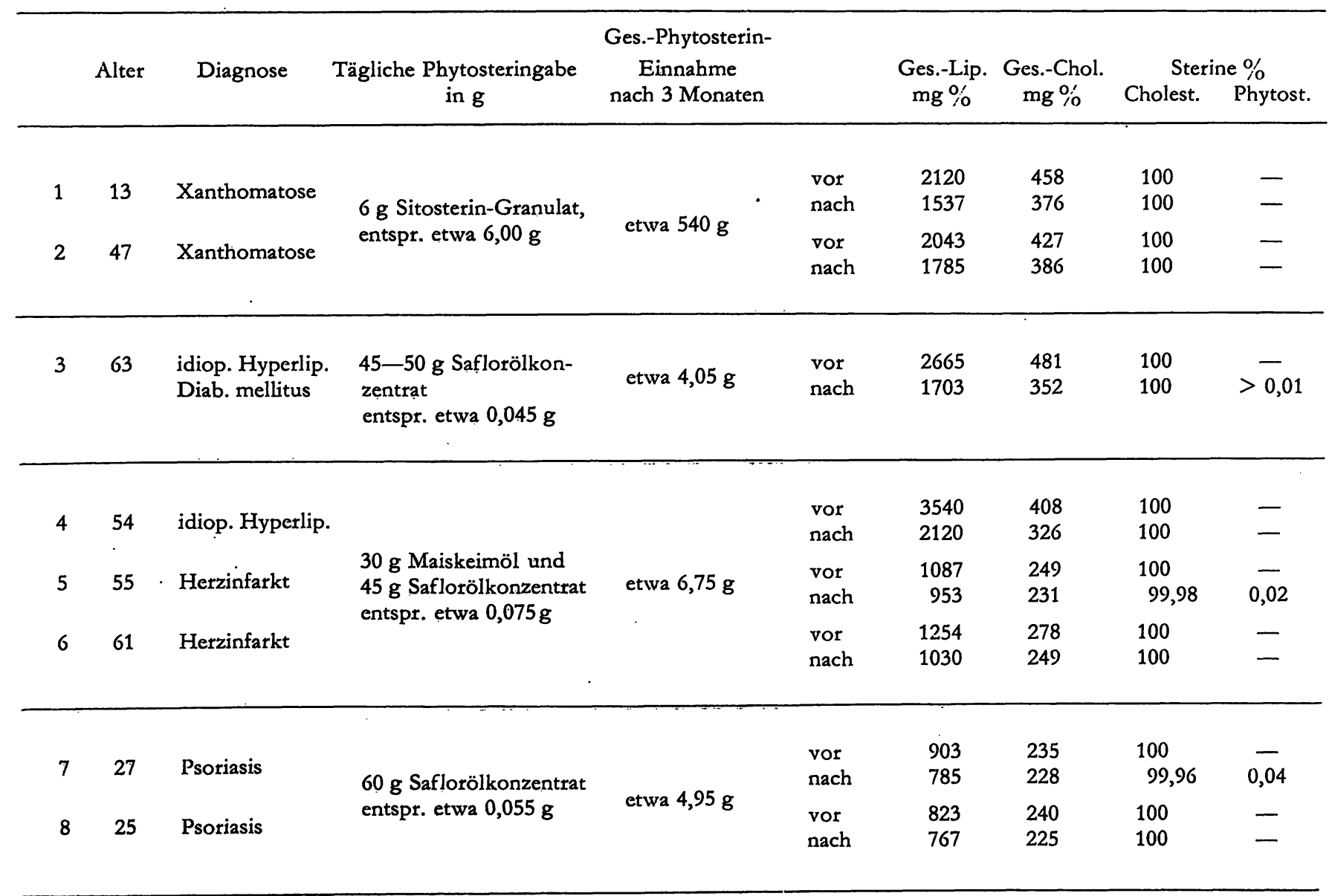



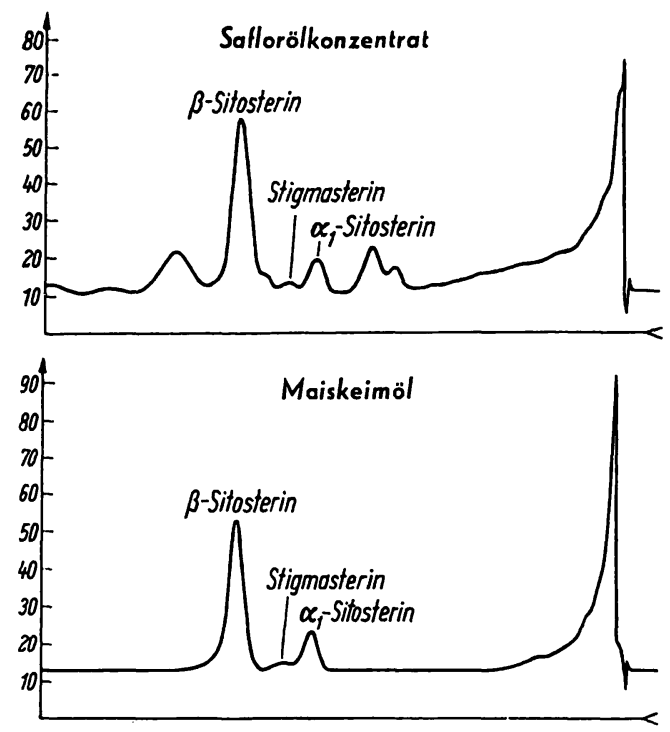

Abb. 5

Gaschromatographische Analyse der Phytosterine von Maiskeimöl und Saflorölkonzentrat

Zusammensetzung

\begin{tabular}{|c|c|c|c|}
\hline \multicolumn{2}{|c|}{ Maiskeimöl } & \multicolumn{2}{|c|}{ Saflorölkonzentrat } \\
\hline [Gesamt-Sterine & $0,088 \%]$ & [Gesamt-Sterine & $0,090 \%]$ \\
\hline$\alpha_{1}$-Sitosterin & $18,0 \%$ & $\alpha_{1}$-Sitosterin & $7,6 \%$ \\
\hline Stigmasterin & $3,5 \%$ & Stigmasterin & $1,7 \%$ \\
\hline$\beta$-Sitosterin & $78,5 \%$ & $\beta$-Sitosterin & $46,7 \%$ \\
\hline & & $\begin{array}{l}\text { übrige, nicht identi- } \\
\text { fizierte Sterine }\end{array}$ & $44,0 \%$ \\
\hline
\end{tabular}

Insgesamt läßt sich sagen, daß eine weitgehende Unabhängigkeit zwischen der Senkung erhöhter oder auch normaler Blutlipidspiegel durch diätetische Maßnahmen und der Resorption pflanzlicher Sterine, sofern überhaupt eine Aufnahme dieser Verbindungen erfolgt, besteht.

\section{Diskussion}

Wenn man unsere Untersuchungsergebnisse auszuwerten versucht, so kann zunächst festgestellt werden, daß der Stoffwechsel pflanzlicher Sterine beim Menschen offenbar komplizierteren Regulationen unterliegt, als bisher angenommen wurde. Angesichts der Tatsache, $\mathrm{da} ß$ bei einem Teil unserer unbehandelten Probanden kleinere Mengen an Phytosterinen im Blut vorhanden waren, erscheinen die früheren Vorstellungen über die Unfähigkeit des menschlichen Organismus, pflanzliche Sterine zu resorbieren, fraglich. Es muß demgegenüber in Betracht gezogen werden, daß unter den bei uns üblichen Ernährungsbedingungen ein Teil der mit den Grundnahrungsmitteln zugeführten Phytosterine die • Darmwand passieren und wie andere Fette und Lipoide ins Blut gelangen. $\mathrm{Ob}$ die Resorption der pflanzlichen Verbindungen in ähnlicher Weise erfolgt, wie die des Cholesterins, d. h. vorwiegend in veresterter Form $(30,47,48,49)$, ist noch unklar.

Wir konnten weiterhin zeigen, daß der Gehalt des Blutes an pflanzlichen Sterinen von Mensch zu Mensch erheblich variiert. Unter den 31 Fällen unseres Unter- suchungsgutes hatten etwas mehr als ein Drittel keine meßbaren Phytosterinanteile im Unverseifbaren des Nüchternserums. Bei den anderen 19 Probanden fanden sich Werte zwischen 0,05\% und 6,47\% Gesamt-Phytosterine. Eine eindeutige Relation zwischen der Höhe des Gesamt-Lipid- bzw. Cholesterinspiegels und dem Phytosteringehalt des Blutserums ergab sich dabei nicht. Allerdings wiesen die gesunden Probanden mit einer Normolipidämie durchweg höhere Gesamt-Phytosterinanteile im Unverseifbaren des Blutserums auf als die Patienten mit einer Blutfettvermehrung. Im Einzelfall wurden aber gelegentlich auch bei hohen GesamtLipid- und Cholesterinwerten verhältnismäßig hohe Phytosterinanteile beobachtet. Diese Unterschiede dürften am ehesten Aușdruck differenter Ernährungsgewohnheiten sein, wobei dem Phytosteringehalt der pflanzlichen Fette und Öle eine Bedeutung zukommt.

Auffälllg ist weiterhin die Tatsache, daß die prozentualen Anteile der einzelnen Phytosterinfraktionen des Blutes von Mensch zu Mensch stark variieren. Hierfür sind entweder. Unterschiede in der Phytosterinzusammensetzung verschiedener Nahrungsbestandteile oder aber eine unterschiedliche Resorption dieser Verbindungen verantwortlich zu machen. Aus tierexperimentellen Untersuchungen wissen wir lediglich, dạß die Beeinflussung der Cholesterinresorption aus dem Darm durch pflanzliche und tierische Sterine uneinheitlich ist (50). Über die Resorptionsquote verschiedener Phytosterine liegen jedoch keine Beobachtungen vor. Hier konnten wir verschiedene Fragen einer Klärung näher bringen: In unseren Belastungsversuchen hat sich gezeigt, daß nach einmaliger peroraler Zufuhr von $24 \mathrm{~g}$ pflanzlicher Sterine bei allen Probanden eine kleinere Menge dieser Verbindungen zur Resorption gelangte und im Blutserum nachgewiesen wurde. Gemessen an der Zunahme der prozentualen Phytosterinanteile, die zwischen 0,02\% und 2,77\% variierten, ergibt sich eine außerordentlich differente Resorptionsquote bei den verschiedenen Probanden. Zweifellos lassen sich dabei Beziehungen zur Applikationsform nachweisen. Nach Verabfolgung der Suspension stieg der Phytosteringehalt des Blutserums im allgemeinen stärker an als nach Einnahme des Granulats. Zu ähnlichen Ergebnissen gelangten auch Schön und Henning (41) mit Hilfe der eingangs erwähnten Bilanzversuche.

Diese Beobachtungen werden freilich durch die Tatsache kompliziert, daß die Resorption der verschiedenen Sterine unterschiedlich erfolgt. Aus den Verlaufskurven in Abbildung 4 geht hervor, daß nach einmaliger Belastung mit pflanzlichen Sterinen sowohl das Ausmaß der prozentualen Zunahme als auch der Zeitpunkt der stärksten Vermehrung der drei Phytosterinfraktionen im Blut different ist. In allen Versuchen war, entsprechend dem hohen $\beta$-Sitosterinanteil beider Präparate die Zunahme dieser Fraktionen im Blutserum unserer Probanden am deutlichsten. 5 der 8 Versuchspersonen zeigten ein Maximum des $\beta$-Sitosterinanstieges nach 5 Stunden p. c. In den anderen drei Fällen wurde eine weitere Zunahme bis zur zehnten Stunde beobachtet. 
Demgegenüber erwies sich die Steigerung des $\alpha_{1}$ Sitosterins mit einem Maximum nach 5 Stunden als durchweg gering, obgleich mit der Emulsion auch beträchtliche Anteile dieses Sterins zugeführt wurden. Lediglich bei einem Probanden kam es zu einem stärkeren $\alpha_{1}$-Sitosterinanstieg. Bemerkenswert ist schließlich die in einzelnen Fällen ausgesprochen starke Zunahme des Stigmasterinspiegels im Blutserum, die um so auffälliger ist, als die peroral zugeführten Stigmasterinmengen außerordentlich gering waren. Wir haben hierfür zunächst noch keine befriedigende Erklärung. Es muß offen bleiben, ob die geringen Stigmasterinanteile der verabfolgten Emulsion selektiv zur Resorption gelangten oder ob bei der Resorption durch Dehydrierung an $\mathrm{C}_{22}$ bzw. $\mathrm{C}_{23}$ unter Bildung einer Doppelbindung ein Teil des $\beta$-Sitosterins in Stigmasterin umgewandelt wurde. Hierfür spricht allerdings die Beobachtung, daß nach Gabe des Granulats, welches keine meßbaren Stigmasterinanteile enthält, bei 2 der 3 Versuchspersonen eine Zunahme dieser Fraktion im Blut nachweisbar war. Uberhaupt ist nach unseren Untersuchungsergebnissen anzunehmen, daß derartige Dehydrierungs- bzw. Hydrierungsprozesse bei der Darmwandpassage oder bereits vorher infolge enteralbakterieller Umbauvorgänge in Anbetracht der engen strukturellen Verwandtschaft der drei verabfolgten pflanzlichen Sterine auftreten. Auch die relativ geringe Zunahme des $\alpha_{1}$-Sitosterins im Blut nach Belastung mit der Emulsion kann darauf zurückgeführt werden, daß ein Teil dieses Sterins durch Hydrierung an $\mathrm{C}_{8}$ bzw. $\mathrm{C}_{9}$ in $\beta$-Sitosterin umgewandelt wurde.

Zwischen den einmaligen Phytosterinbelastungen und den 10-Tagesversuchen, bei denen täglich $18 \mathrm{~g}$ pflanzliche Sterine verabfolgt wurden, ergaben sich gewisse Parallelen. Unter den Bedingungen einer länger dauernden Applikation nahm wiederum in erster Linie der $\beta$-Sitosteringehalt des Blutserums bei unseren Probanden $z u$, deutlicher und schneller nach Einnahme der Emulsion als nach Zufuhr des Granulats. Insgesamt weniger ausgeprägt war demgegenüber der Anstieg der anderen Phytosterinfraktionen, wenn auch in Einzelfällen eine starke Vermehrung von $\alpha_{1}$-Sitosterin bzw. Stigmasterin beobachtet werden konnten. Wichtig ist die Tatsache, $\mathrm{da} ß$ der Gesamt-Phytosteringehalt des Blutes bei 3 Versuchen mit der Emulsion nach 5 Tagen im allgemeinen die höchsten Werte erreichte. Ein weiterer Konzentrationsanstieg wurde dabei nicht angetroffen. Unsere Ergebnisse stehen in Übereinstimmung mit den Befunden von Gould (40), der mit Hilfe von markiertem $\beta$-Sitosterin bei einer Patientin den Höhepunkt der spezifischen Aktivität des Blutes bereits nach 3 Tagen feststellte. Diese Beobachtungen sprechen dafür, daß bei regelmäßiger Zufuhr größerer Mengen pflanżlicher Sterine der resorbierte und ausgeschiedene Anteil dieser Verbindungen annähernd gleich groß ist. Gleichzeitig dokumentiert sich darin eine Unabhängigkeit der Phytosterinresorptionsrate von der Wirkung des Sitosterins auf die Cholesterinresorption und damit auf die blutcholesterinsenkende Wirkung dieser Verbindungen.
$\mathrm{Zu}$ ähnlichen Vorstellungen gelangte man auch angesichts unserer Untersuchungsergebnisse an 8 Probanden, denen über drei Monate pflanzliche Sterine entweder als Granulat oder ihm Rahmon einer diätetischen Therapie mit polyensäurenreichen Ölen zugeführt wurden. Es erscheint besonders wichtig, daß bei den beiden Xanthomatosekranken die Applikation von mehr als $500 \mathrm{~g}$ Sitosterin zwar zu einer Senkung des Blutcholesterins und Blutlipidspiegels führte, daß es dabei jedoch nicht zum Auftreten meßbarer Phytosterinanteile im Serum kam. Aus Bilanzversuchen von Schrade (51) ist bekannt, daß bei der Xanthomatose die Fähigkeit zur Resorption größerer Cholesterinmengen stark herabgesetzt ist. Unsere Untersuchungen sprechen in gleicher Weise für eine praktisch aufgehobene Phytosterinresorption solcher Patienten, obwohl nach den Blutanalysen der blutcholesterin- und lipidsenkende Effekt peroraler Sitosteringaben evident ist. Auch die Zufuhr pflanzlicher Öle führte sowohl bei pathologischen Hyperlipidämien als auch bei 2 Psoriasiskranken mit einer Normolipidämie nicht zur Resorption nennenswerter Phytosterinanteile. Allerdings sind die im Rahmen eines solchen Diätregimes aufgenommenen Mengen pflanzlicher Sterine relativ gering. Man muß dennoch annehmen, daß auch in diesen Fällen Anomalien der Sterinresorption, wie bei der Xanthomatose, vorhanden sind.

Insgesamt läßt sich aus diesen Ergebnissen die Folgerung ableiten, daß bei bestimmten Fettstoffwechselstörungen die Wirkung pflanzlicher Sterine auf den Blutcholesterinspiegel keineswegs dadurch zustande kommt, daß diese pflanzlichen Verbindungen resorbiert werden und den intermediären Cholesterinstoffwechsel beispielsweise im Sinne einer Synthesehemmung beeinflussen. Inwieweit diese Vorstellungen auch für die Beeinflussung normaler oder leicht erhöhter Blutlipide zutreffen, sei dahingestellt. Wir haben zwar Anhaltspunkte dafür, daß zwischen der Höhe des Phytosterinspiegels im Blut und dem Gehalt an Gesamt-Lipiden und Cholesterin eine gewisse Relation besteht, es überwiegen jedoch nach unseren Untersuchungen die Argumente, die für einen enteralen die Cholesterinresorption hemmenden Mechanismus des Phytosterins sprechen. Die Wirkung resorbierter Sterine auf den intermediären Cholesterinstoffwechsel dürfte nur als Teilfaktor in Betracht kommen. Wir stehen hier allerdings erst am Anfang unserer Erkenntnisse. Es ist möglich, daß sich aus Bilanz- und Resorptionsversuchen mit den verschiedensten Phytosterinen weitere Aufschlüsse über den Sterinstoffwechsel des Menschen und vielleicht neue Konsequenzen für eine cholesterin- und lipidsenkende Therapie mit diesen Substanzen ergeben. Wenn beispielsweise die Zufuhr polyensäurenreicher Öle über den lipidsenkenden Effekt hinaus zur Exkretion großer Sterinmengen führt, deren Zusammensetzung $z$. T. noch unbekannt ist $(46,52,53)$, so sollte diese Feststellung Anlaß sein, dem Sterinstoffwechsel des Menschen nicht nur, wie bisher von der quantitativen, sondern auch von der qualitativen Seite her Rechnung zu tragen. 


\section{Literatur}

1. Peterson, D. W., Proc. Soc. exp. Biol. Med. 78, 143 (1951). 2. Pollak, O. J., Circulation (New York) 7, 702 (1953). 3. Betzien, G., H. Bracharz, P. B. Diezel, H. Franke, R. Kufin und Th. SeIdL, Arzneimittel-Forsch. 11, 751 (1961). - 4. BARBER, J. M. und A. P. GrANt, Brit. Heart J. 17, 296 (1955). - 5. Berge, K. G., R. W. P. Achor, N. W. Barker und M. H. Power, Amer. Heart J. 58, 849 (1959). - 6. BEST, M. M., C. H. DunCAN, E. vaN Loon und J. D. WATHEN, Circulation (New York) 10, 201 (1954). - 7. Best, M. M. und C. H. Duncan, Ann. Int. Med. 45, 614 (1956). - 8. Best, M. M., C. H. Duncan, E. J. van Loon und J. D. Wathen, Amer. J. Med. 19, 61 (1955). - 9. Farquhar, J. W. und M. Solokow, Circulation (New York) 17, 890 (1958). 10. Joyner, C. R. und P. T. Kuo, Circulation (New York) 10, 589 (1954). - 11. JoYner JR., C. und P. T. Kuo, Amer. J. Med. Sc. 230, 636 (1955). - 12. KaEgr, P. und F. Koller, Helvet. med. acta 4, 392 (1957). - 13. Lesesne, J. M., C. W. CAstor und S. W. Hoobler, Univ. Michigan Med. Bull. 21, 13 (1955). 14. Rrley, F. P. und A. Sterner, Circulation (New York) 16, 723 (1957). - 15. SACHS, B. A. und R. E. Weston, Arch. Int. Med., Chicago 97, 738 (1956). - 16. FarQuhar, J. W., R. E. SMITH und M. E. Dempsey, Circulation (New York) 14, 77 (1956). 17. Shipley, R. E., Trans. N. Y. Acad. Sci. Ser. II 18, 111 (1955). 18. Levere, A. H., R. C. Bozian, G. Craft und R. S. JaCkson, Metabolism, 7, 338 (1958). - 19. WILKInson, C. F., E. Boyle, R. S. Jackson und M. R. Benjamin, Metabolism 4, 302 (1955). 20. Friedman, M., R. Homer und S. O. Byers, Circulation (New York) 12, 709 (1955). - 21. Pomeranze, J. und M. Chessin, Amer. Heart J. 49, 262 (1955). - 22. WILKINSON, C. F., E. BoyLE, R. S. JACKson und M. R. Benjamin, Metabolism 4, 302 (1955). 23. Hernandez, H. H., D. W. Peterson, I. L. Chatkoff und W. G. Dauben, Proc. Soc. exp. Biol. Med. 83, 498 (1953). 24. Hernandez, H. H. und I. L. Chaikoff, Proc. Soc. exp. Biol. Med. 87, 541 (1954). - 25. Beveridge, J. M. R., W. F. Connell und G. A. Mayer, Federat. Proc. 16, 11 (1957). - 26. Davis, W. W., Trans. New York Acad. Sc. 18, 123 (1955). - 27. Hudson, J. L., E. R. Diller, R. R. Pfeiffer und W. W. Davis, Proc. Soc. exp. Biol. Med. 102, 461 (1959). - 28. Pererson, D. W., E. A. Shineour, N. F. Peek und H. W. Gaffey, J. Nutrit. 50, 191
(1953). - 29. Best, M. M. und C. H. Duncan, J. Nutrit. 65, 169 (1958). - 30. Chajkoff, I. L., B. Bloom, M. D. Siperstein, J. Y. Kiyasu, W. O. Reinfeardt, W. G. Dauben und J. F. EastHAM, J. biol. Chemistry 194, 407 (1952). - 31. SсHÖNHEIMER, R., Hoppe-Seyler's Z. physiol. Chem. 180, 24 (1929). - 32. ScнöNhermer, R., H. von Behring, H. Hummel und L. Schinder. Hoppe-Seyler's Z. physiol. Chem. 192, 75 (1930). - 33. SсHÖNheimer, R., H. von Behring und R. Hummel, Hoppe-Se yler's Z. physiol. Chem. 192, 117 (1930). - 34. SCHÖNHEIMER, R., Hoppe-Seyler's Z. physiol, Chem 192, 77 (1930). - 35. SснӧNhermer, R. und H. von BeHRing, Klin. Wschr. 9, 1308 (1930). 36. Dam, H. U. und U. Starup, Biochem. Z. 274, 117 (1934). 37. Shipley, R. E., R. R. Pfeiffer, M. M. Marsh und R. C. Anderson, Circulation (New York) 6, 353 (1958). - 38. Swelc, L., T. Boiter, H. Field JR. und C. R. Treadwell, Proc. Soc. exp. Biol. Med. 86, 295 (1954). - 39. Goulp, R. G., L. V. Lotz und E. M. LiLLY, Proceedings of 2nd International Conference on the Biochemical Problems of Lipids, Butterworths Scientific Publ. 56, S. 353 (1955). - 40. Gould, R. G., Trans. New York Acad. Sc. 18, 129 (1955). - 41. SCHÖN, H. und N. HENning, Dtsch. med. Wschr. 84, 1385 (1959). - 42. SCHÖN, H. und E. ENGELHARDT, Arzneimittel-Forsch. 10, 491 (1960). - 43. Bloor, W. R., J. biol. Chemistry 17, 377 (1914): - 44. SchöNHEIMER, R. und W. M. SPERry, J. biol. Chemistry 106, 745 (1934). - 45. SPERRY, W. M. und M. WeBb, J. biol. Chemistry 187, 97 (1950). - 46. BeErthuis, R. K. und J. H. Recourt, Colloques Internationaux du Centre National de la Recherche Scientifique XCIX Marseille 18-20 Juillet 1960, S. 206, Centre National de la Recherche Scientifique, Paris (1961). - 47. Best, M. M., C. H. Duncan, E. J. van Loon und J. D. Wathen, Amer. J. Med. 19, 61 (1955). - 48. BollMAN, J. L. und E. V. Flock, Amer. J. Physiol. 164, 481 (1951). 49. Swell, L., H. Field und C. R. Treadwell, Proc. Soc. exp. Biol. Med. 103, 263 (1960). - 50. Daskalakis, E. G. und I. L. Chatkoff, Arch. Biochem. Biophysics 58, 373 (1955). - 51. Schrade, W., Erg. inn. Med. 62, 743 (1943). - 52. Gordon, H., B. Lewis, L. Eales und E.F. Brocks, Lancet, 2, 1299 (1957). 53. Beerthuis, R. K., Persönliche Mitteilung (1961).

Priv. Doz. Dr. med. Eberhard Böhle I. Medizinische Universitäts-Klinik 6 Frankfurt/Main, Ludwig-Rehn-Str. 14 